\title{
Selective lesions of medial septal GABAergic neurons disrupt Hippocampal Theta Rhythm Amplitude
}

\author{
Miriam G Perez-Cordova ${ }^{1,2}$, Saul Jaime ${ }^{1,2}$, Herminio Guajardo ${ }^{1,2}$, Luis V Colom ${ }^{1,2^{*}}$ \\ From Nineteenth Annual Computational Neuroscience Meeting: CNS*2010 \\ San Antonio, TX, USA. 24-30 July 2010
}

Septal inputs critically modulate hippocampal function. However, the role of Medial Septal/Diagonal Band (MS/ DB) neuronal populations in hippocampal theta rhythm generation is still under debate. MS/DB GABAergic neurons selectively innervate inhibitory hippocampal interneurons and through this action exert a powerful control on hippocampal excitability. To further investigate the role of the MS/DB GABAergic neurons in hippocampal theta rhythm generation, the antibody Gamma amino butyric acid Transporter 1 (GAT-1) conjugated to saporin (SAP, $3 \mu \mathrm{L}$ at $325 \mathrm{ng} / \mu \mathrm{L}$ ) was injected in the MS/DB of adults Sprague Dawley rats to selectively destroy GABAergic neurons. Population estimates of MS/DB GABAergic neurons were analyzed using StereoInvestigator 9. A significant reduction $(75 \%$, $p<0.0001)$ in the MS/DB GABAergic population was observed, while the cholinergic and glutamatergic neuronal population were spared. The effects of this GABAergic neuronal loss in hippocampal function were investigated by hippocampal EEG recordings. EEG recordings of freely moving rats were acquired using the Harmonie Stellate monitoring system. EEGs were recorded before and after the intraseptal injection of anti-GAT-1-SAP. Two second duration trace, representative of theta I and theta II were transferred off line to ClampFit 9 software to measure amplitude and produce a power spectrum. After analysis of the theta traces the rat brains were sliced and stereology was once again performed to determine the relationship between the destruction of MS/DB GABAergic neurons and the hippocampal theta rhythm.

\footnotetext{
* Correspondence: Luis.Colom@utb.edu

${ }^{1}$ Biological Science, University of Texas at Brownsville and Texas Southmost College, Texas, 78520, USA
}

\section{Conclusions}

A significant reduction between the baseline and experimental recording, in amplitude $(30 \%)$ in both theta I and theta II $(p<0.001)$ were observed, while the frequencies were not statistically significant. No abnormal activity in these traces (i.e. gamma waves) was observed. Our results illustrate that the observed reduction of theta rhythm was always associated with 75\% loss of MS/DB GABAergic neurons. Thus, MS/BD GABAergic neurons play an important role in theta rhythm amplitude modulation. Ongoing studies are dedicated to determine if there is a correlation between destruction of a wider area of the MS/DB GABAergic neurons and its subsequent functional changes in hippocampal theta rhythm.

\section{Acknowledgments \\ Funded by NIH grant SC1NS065386 to Luis V Colom.}

\section{Author details}

'Biological Science, University of Texas at Brownsville and Texas Southmost College, Texas, 78520, USA. ${ }^{2}$ Center for Biomedical Studies, University of Texas at Brownsville and Texas Southmost College, Texas, 78520, USA.

\section{Published: 20 July 2010}

\section{References}

1. Colom LV, Garcia-Hernandez A, Castaneda MT, Perez-Cordova MG, GarridoSanabria ER: Septo-Hippocampal Networks in Chronically Epileptic Rats: Potential Antiepileptic Effects of Theta Rhythm Generation. 2006.

2. Pang $\mathrm{KCH}$, Jiao $X$, Servatius RJ: Role of medial septal neurons in learning and extinction Effects of the novel GABA immunotoxin GAT 1-SAP. Targeting Trends 2008.

doi:10.1186/1471-2202-11-S1-P125

Cite this article as: Perez-Cordova et al: Selective lesions of medial septal GABAergic neurons disrupt Hippocampal Theta Rhythm Amplitude. BMC Neuroscience 2010 11(Suppl 1):P125. 